\title{
5 Spezialgesetzliche Einschränkungen der Sekundärnutzung
}

Gibt es spezielle Behandlungsdaten, die aufgrund spezialgesetzlicher Regelungen hinsichtlich ihrer Sekundärnutzung eingeschränkt oder für die besondere Rahmenbedingungen zu berücksichtigen sind? Welche Regelungen sind das und welches sind die zu berücksichtigenden Rahmenbedingungen?

\subsection{Bundeseinheitliche Regelungen}

\subsubsection{Gendiagnostikgesetz (GenDG)}

Genetische Informationen weisen im Vergleich zu konventionellen medizinischen Daten eine Reihe von Besonderheiten auf. Durch genetische Untersuchungen können auch zukünftige Krankheiten erkannt werden, die noch nicht ausgebrochen sind, was zu weitreichenden Konsequenzen für die Betroffenen führen kann. Außerdem ist anhand genetischer Daten nicht nur eine Aussage über die untersuchte Person selbst, sondern eventuell auch über Dritte (Blutsverwandte) möglich. Um diesen verschiedenen Aspekten Rechnung zu tragen, regelt das Gendiagnostikgesetz den Umgang mit genetischen Informationen in spezieller Weise. 159 \$ 1 S. 1 GenDG erklärt den Schutz der Menschenwürde und insbesondere des Rechts auf informationelle Selbstbestimmung zum Hauptziel dieses Gesetzes.

159 Vgl. Bundestags-Drucksache 16/10532, S. 1 ff. 
Eine weitere Besonderheit genetischer Daten ist, dass durch sie ein genetischer Fingerabdruck einer Person erstellt werden kann, wodurch diese auch über weitere Verarbeitungsschritte hin eindeutig identifizierbar bleibt. ${ }^{160}$ Die genetischen Merkmale des Betroffen lassen sich somit kaum von dessen Identität trennen, was eine echte Anonymisierung ausschließt oder zumindest erheblich erschwert. ${ }^{161}$

Für die Ergebnisse einer genetischen Untersuchung soll deshalb ein besonders hoher Geheimnisschutz gelten und nur die betroffene Person soll bestimmen dürfen, was mit den entsprechenden Daten geschieht. ${ }^{162}$ Daher schützt das Gendiagnostikgesetz genetische Daten in einem stärkeren Maße als sonstige personenbezogene Daten, selbst Gesundheitsdaten, durch andere Datenschutzvorschriften geschützt werden. ${ }^{163}$

Da die Vorschriften des Gendiagnostikgesetzes diesen erhöhten Schutz umsetzen sollen, genießen sie Vorrang vor anderen Datenschutznormen. ${ }^{164}$ Das BDSG oder andere allgemeinere Vorschriften über den Datenschutz finden insofern nur subsidiär Anwendung, also nachrangig bzw. zur Lückenfüllung, sofern das GenDG keine speziellen Regelungen enthält (vgl. $\mathbb{S} 1$ Abs. 3 S. 1 BDSG). ${ }^{165}$ Würde beispielsweise eine Erlaubnisklausel des BDSG oder eines Landeskrankenhausgesetzes die Forschung mit Gendaten an sich erlauben, das GenDG diese aber von der Einwilligung des Patienten abhängig machen, so würde sich das Einwilligungserfordernis des GenDG durchsetzen. ${ }^{166}$

\subsubsection{Anwendung des Gendiagnostikgesetzes bei der Sekundärnutzung von Patientendaten zu Forschungszwecken}

Ob die speziellen Vorschriften des Gendiagnostikgesetzes bei der Sekundärnutzung von Patientendaten zu beachten sind, hängt zunächst davon ab, ob das Gendiagnostikgesetz für solche Sachverhaltskonstellationen Anwendung findet. Gemäß $\mathbb{2}$ Abs. 1 GenDG gilt das Gendiagnostikgesetz für genetische Untersuchungen und im Rahmen genetischer Analysen bei geborenen Menschen sowie bei Embryonen und Föten während der Schwangerschaft und den Umgang mit dabei gewonnenen genetischen Proben und genetischen Daten bei genetischen Untersuchungen zu medizinischen Zwecken, zur Klärung der Abstammung sowie im Versicherungsbereich und im Arbeitsleben.

Genetische Daten werden in $\mathbb{3} 3 \mathrm{Nr}$. 11 GenDG legaldefiniert als die durch eine genetische Untersuchung oder die im Rahmen einer genetischen Untersuchung durchgeführten genetischen Analyse gewonnenen Daten über genetische Eigenschaften. Genetische Daten sind somit Daten, die zu medizinischen Zwecken, in der Regel zur Untersuchung eines bestehenden oder vermuteten veränderten Gesundheitszustandes, erhoben werden. ${ }^{167}$ Unter Umgang mit genetischen Daten ist jede Erhebung, Verarbeitung und Nutzung genetischer Daten zu verstehen, ${ }^{168}$ insbesondere aber eine

\footnotetext{
160 Vossenkuhl, Der Schutz genetischer Daten unter besonderer Berücksichtigung des Gendiagnostikgesetzes, S. 97.

161 Zur Frage, wie weit dieser Ansatz reicht, s.u. S. $57 f$.

162 Schillhorn/Heidemann, GenDG, $\$ 11$ Rdnr. 8.

163 Vossenkuhl, Der Schutz genetischer Daten unter besonderer Berücksichtigung des Gendiagnostikgesetzes, S. 95

164 Schillhorn/Heidemann, GenDG, §11 Rdnr. 9.

165 Insofern besteht auch ein Vorrang des GenDG vor den Landeskrankenhaus- oder anderen Landesgesetzen.

166 Zur vorgelagerten Frage, ob und ggf. inwieweit das GenDG überhaupt für die Forschung mit Gendaten gilt und damit ein entsprechendes Einwilligungserfordernis etablieren kann, s. sogleich S. 54 .

167 Schillhorn/Heidemann, GenDG, \) 2 Rdnr. 11.

168 In Anlehnung an die Terminologie des BDSG (dort v.a. $\$ 1$ Abs. 1 in Verbindung mit $\$ 3$ Abs. 3, 4, 5).
} 
Verwendung dieser Daten zu anderen Zwecken, als zu denen sie ursprünglich erhoben wurden. ${ }^{169}$ Werden die Daten zunächst beispielsweise zur Diagnose oder Behandlung einer Erbkrankheit erhoben und anschließend sekundär für Forschungszwecke genutzt, liegt ein Umgang mit genetischen Daten vor.

Fraglich ist jedoch, ob das Gendiagnostikgesetz überhaupt bei der Sekundärnutzung der Daten zu Forschungszwecken Anwendung findet, denn für die Forschung soll das Gendiagnostikgesetz nach $\$ 2$ Abs. 2 Nr. 1 GenDG nicht gelten. Hier soll es bei der Anwendung der allgemeinen gesetzlichen Regelungen, insbesondere des AMG und MPC bleiben. ${ }^{170}$ Der Ausschluss der Anwendung bei genetischen Daten zu Forschungszwecken wird vielfach kritisiert. ${ }^{171}$ Unter Forschung versteht man im Rahmen der Ausnahme in $\$ 2$ Abs. 2 Nr. 1 GenDG nicht nur Grundlagenforschung, sondern auch angewandte Forschung im Sinne eines weiten Forschungsbegriffs. ${ }^{172}$ Der Umgang mit genetischen Proben und Daten zu Forschungszwecken sollte nach dem ursprünglichen Willen des Gesetzgebers ausdrücklich ausgeklammert sein, da die Einzelheiten hierzu im noch zu schaffenden Biobankengesetz geregelt werden sollten. ${ }^{173}$

Von der Ausnahme sind jedoch nur solche genetischen Daten erfasst, die ausschließlich zu Forschungszwecken gewonnen werden. ${ }^{174}$ Zwischen medizinischen Zwecken und Forschungszwecken kann es in der Praxis zu Überschneidungen kommen, wenn primär für medizinische Zwecke gewonnene genetische Daten später für Forschungszwecke genutzt werden. ${ }^{175}$ Das Gendiagnostikgesetz findet auch für den umgekehrten Fall Anwendung, wenn genetische Untersuchungen ursprünglich zur Forschung durchgeführt worden sind, sich aber in deren Verlauf zeigt, dass die Daten auch medizinische Relevanz für die Behandlung des betroffenen Probanden bzw. Patienten haben. ${ }^{176}$ Wenn von vornherein sowohl medizinische als auch forschende Zwecke mit der genetischen Untersuchung verfolgt werden, überwiegt der medizinische Aspekt, sodass die Vorschriften des Gendiagnostikgesetzes beachtet werden müssen. ${ }^{177}$ Auch auf eine spätere Sekundärnutzung von Gendaten, die ursprünglich im Rahmen einer Untersuchung für medizinische Zwecke gewonnen wurden, zu Forschungszwecken findet das Gendiagnostikgesetz Anwendung. ${ }^{178}$ Dies gilt sowohl für die ursprüngliche medizinische Verwendung als auch für die anschließende Verwendung zu Forschungszwecken. ${ }^{179}$

Für rein zu genetischen Forschungszwecken gewonnenen Daten bleibt es bei der Anwendung der allgemeinen gesetzlichen Bestimmungen des Datenschutzrechts nach dem BDSG bzw. den bereichsspezifischen Datenschutzbestimmungen der Länder. ${ }^{180}$

169 Kern, in: Kern (Hg.), GenDG, $\$ 2$ Rdnr. 4.

170 Kern, in: Kern (Hg.), GenDG, \ 2 Rdnr. 10.

171 Vossenkuhl, Der Schutz genetischer Daten unter besonderer Berücksichtigung des Gendiagnostikgesetzes, S. 111f.; Kern, in: Kern (Hg.), GenDG, § 2 Rdnr. 12.

172 Sosnitza/Op den Camp, MedR 2011, 401, 402.

173 Fenger, in: Spickhoff (Hg.), Medizinrecht, GenDG, \$ 2 Rdnr. 1. Nachdem das GenDG in der 16. Legislaturperiode unter einer Großen Koalition beschlossen wurde, wurden in der 17. Legislaturperiode entsprechende Vorstöße in Richtung eines Biobankengesetzes jedoch von einer schwarz-gelben Koalition im Bundestag abgelehnt, Drucksache 17/8873 v. 06.03.2012.

174 Schillhorn/Heidemann, GenDG, \2 Rdnr. 17.

175 Schillhorn/Heidemann, GenDG, $\$ 2$ Rdnr. 9.

176 Schillhorn/Heidemann, GenDG, \ 2 Rdnr. 19.

177 Schillhorn/Heidemann, GenDG, \& 2 Rdnr. 20.

178 Kern, in: Kern (Hg.), GenDG, § 2 Rdnr. 4.

179 Dies gilt nach hier vertretener Auffassung gerade auch dann, wenn die spätere Forschungsnutzung nicht von vornherein intendiert war.

180 Bundestags-Drucksache 16/10532, S. 20. 


\subsubsection{Vorgaben des Gendiagnostikgesetzes für den Umgang mit genetischen Daten}

Da das Gendiagnostikgesetz bei der Sekundärnutzung von Behandlungsdaten Anwendung findet, müssen die speziellen Vorgaben des Gendiagnostikgesetzes beim Umgang mit den genetischen Daten beachtet werden.

\section{Aufklärungserfordernis}

$\mathbb{S} 9$ Abs. 2 Nr. 3 GenDG schreibt vor, dass der Patient vor der Erteilung seiner Einwilligung insbesondere auch über die vorgesehene Verwendung der genetischen Probe sowie der Untersuchungs- oder der Analyseergebnisse umfassend aufzuklären ist. In diesem Zusammenhang muss der Betroffene auch über die Möglichkeit einer weiteren Verwendung der Daten zu Zwecken wissenschaftlicher Forschung aufgeklärt werden. ${ }^{181}$ Eine eventuelle später beabsichtigte Nutzung der Daten zu Forschungszwecken muss dem Patienten somit vorab mitgeteilt werden, um dem Aufklärungserfordernis $\mathrm{zu}$ entsprechen. ${ }^{182}$

Nach dieser Aufklärung ist die ausdrückliche und schriftliche Einwilligung der betroffenen Person über den Umfang der genetischen Untersuchung als auch darüber, ob und inwieweit das Untersuchungsergebnis zur Kenntnis zu geben oder zu vernichten ist ( $\mathbb{8}$ Abs. 1 S. 2 GenDG), einzuholen.

\section{Grundsatz der Zweckbindung}

Für genetische Daten gilt der Grundsatz der Zweckbindung, wonach die Ergebnisse der genetischen Untersuchung grundsätzlich nur für den Zweck verwendet werden dürfen, für den sie ursprünglich gedacht waren. Gemäß $\mathbb{S} 13$ Abs. 1 S. 1 GenDG darf eine genetische Probe nur für die Zwecke verwendet werden, für die sie gewonnen worden ist. $\mathbb{1 1}$ Abs. 3 GenDG stellt die Weitergabe des Ergebnisses einer genetischen Untersuchung unter den ausdrücklichen Einwilligungsvorbehalt des Betroffenen, sodass diese Vorschrift als Parallelnorm zu $₫ 13$ Abs. 1 S. 1 gleichfalls eine Bindung der genetischen Daten vorschreibt. ${ }^{183}$ Somit dürfen genetische Daten, die zu medizinischen Zwecken erhoben wurden, nur mit Einwilligung des Betroffenen für Forschungszwecke verwendet werden ( $\$ 13$ Abs. 2 GenDG).

\section{Grundsatz: Keine Weitergabe der Ergebnisse an Dritte ohne Einwilligung}

Nach $₫ 11$ Abs. 1 GenDG darf das Ergebnis der genetischen Untersuchung nur der betroffenen Person mitgeteilt werden. $\$ 11$ GenDG dient dem Schutz des informationellen Selbstbestimmungsrechts des Patienten. ${ }^{184}$ Eine mit der genetischen Analyse beauftragte Person oder Einrichtung darf das Ergebnis der genetischen Analyse nur der ärztlichen Person mitteilen, die sie mit der genetischen Analyse beauftragt hat, $\mathbb{} 11$ Abs. 2 GenDG. Für Krankenhäuser soll dies der ärztliche Dienst des Krankenhauses sein, wobei die Kliniken intern festlegen können, dass Ergebnisse beispielsweise nur an das behandelnde Ärzteteam mitzuteilen sind. ${ }^{185}$ Auch hierdurch sollen der Daten-

181 Bundestags-Drucksache 16/10532, S. 27

182 Sollte sich ein Forschungsinteresse erst später ergeben, dann wäre hierfür eine erneute Einwilligung einzuholen.

183 Vossenkuhl, Der Schutz genetischer Daten unter besonderer Berücksichtigung des Gendiagnostikgesetzes, S. 90.

184 Genger, NJW 2010, 113, 115

185 Schillhorn/Heidemann, GenDG, $§ 11$ Rdnr. $5 f$. Eine solche Beschränkung der Mitteilung auf das behandelnde Ärzteteam sollte aus allgemeinen Erwägungen von Datenschutz und Schweigepflicht heraus auch tatsächlich erfolgen. 
schutz und das informationelle Selbstbestimmungsrecht des Patienten vollständig gesichert werden. ${ }^{186}$

Das Ergebnis der genetischen Analyse darf jedoch anderen Personen von der verantwortlichen ärztlichen Person mitgeteilt werden, wenn die ausdrückliche und schriftliche Einwilligung der betroffenen Person vorliegt ( $\$ 11$ Abs. 3 GenDG). Die Sekundärnutzung der (personenbezogenen) genetischen Daten seitens Dritter ist somit (nur) mit einer ausdrücklichen und schriftlichen Einwilligung des Patienten möglich, der dadurch der Verwendung für Forschungszwecke zustimmt ( $\mathbb{1 1} \mathrm{Abs} .3, \mathbb{1} 13 \mathrm{Abs} .2 \mathrm{CenDG}$ ).

Dieses durch das GenDG statuierte Einwilligungserfordernis ist als spezielles Bundesrecht vorrangig gegenüber allgemeinen Forschungserlaubnissen des Bundesrechts wie der in $\mathbb{2} 28$ Abs. 6 Nr. 4 BDSC oder auch spezifischen Forschungsklauseln in den Landeskrankenhausgesetzen, welche unter bestimmten Voraussetzungen die Forschung ohne Einwilligung zulassen. ${ }^{187}$ Der Rückgriff auf solche Erlaubnisnormen kommt bezüglich der Sekundärnutzung personenbezogener Gendaten nicht in Betracht. Allerdings sind die allgemein für die Verarbeitung personenbezogener Daten in diesen allgemeineren Gesetzen geregelten Rahmenbedingungen, wie beispielsweise zu weitergehenden Rechten des Betroffenen auf Auskunft und Berichtigung sowie die Datenschutzkontrolle, auch auf Gendaten anzuwenden, da das GenDG insoweit keine speziellen Regelungen enthält. ${ }^{188}$

\section{Ausnahme: Isolierte Weitergabe des Befundergebnisses}

Nach $\mathbb{1 1}$ Abs. 3 GenDG darf die verantwortliche ärztliche Person das Ergebnis der genetischen Untersuchung oder Analyse, wie gesehen, anderen nur mit ausdrücklicher und schriftlicher Einwilligung der betroffenen Person mitteilen. Wie bereits erwähnt, ist eine vollständige Anonymisierung genetischer Daten aufgrund ihrer Besonderheit (genetischer Fingerabdruck) in der Regel nicht möglich. Deshalb sind genetische Daten durch das Gendiagnostikgesetz geschützt und dürfen ohne Einwilligung des Betroffenen nicht an Dritte weitergegeben werden.

Fraglich ist jedoch, was für Gendaten im weiteren Sinne, die nicht den genetischen Fingerabdruck des Betroffenen beinhalten, sondern letztlich nur einzelne Ergebnisbefunde (z.B. das Vorliegen einer bestimmten Cenmutation) aus der genetischen Untersuchung darstellen, gelten soll.

Konkret geht es um die Frage, ob grundsätzlich alle Daten, die im Rahmen einer genetischen Untersuchung gewonnen werden, dem speziellen Schutz des Gendiagnostikgesetzes unterfallen. Dies richtet sich danach, ob man von einer generellen Exzeptionalität genetischer Informationen ausgeht.

Der Ansatz der genetischen Exzeptionalität geht davon aus, dass sich genetische Informationen „qualitativ und grundsätzlich von anderen medizinischen Informationen unterscheiden “. ${ }^{189}$

186 Schillhorn/Heidemann, GenDG, §11 Rdnr. 4.

187 Zwar sind die genannten Regelungen auch nach der Gesetzesbegründung (Bundestags-Drucksache 16/10532, S. 16) weiterhin auch auf Gendaten anwendbar, allerdings nur soweit das GenDG keine Regelungen trifft, also beispielsweise bezüglich der in der Begründung hervorgehobenen „Schadenersatz-, Auskunfts- und Berichtigungsansprüche der betroffenen Personen“" sowie der „Vorschriften über die Datenschutzkontrolle“.

188 So auch die Gesetzesbegründung, s. soeben Fn. 187.

189 Vgl. Damm/König, MedR 2008, 62ff. 
Eine Meinung in der Literatur ${ }^{190}$ befürwortet diesen Ansatz einer uneingeschränkten Exzeptionalität genetischer Informationen. Demnach sollen auch die Ergebnisse von genetischen Untersuchungen von der genetischen Exzeptionalität umfasst sein. Eine Weitergabe der Befundergebnisse, selbst in anonymisierter Form, ist demnach insbesondere für polygene Erkrankungen, also einer Mutation mehrerer Cene, ausgeschlossen. Begründet wird dies damit, dass selbst bei einzelnen DNA-Teilstücken die Individualität des untersuchten Genoms noch so stark sei, dass eine Identifizierung des Betroffenen möglich ist ${ }^{191}$. Dies führt dazu, dass unter Umständen sogar nur auf Grundlage von Ergebnisdaten ein Rückschluss auf eine bestimmte Person möglich sein kann. ${ }^{192}$ Nach dem vom Gendiagnostikgesetz angestrebten weitreichenden Schutz des informationellen Selbstbestimmungsrechts des Betroffenen verbietet sich dieser Meinung folgend die isolierte Weitergabe aus genetischen Untersuchungen gewonnener Befunde ohne die Einwilligung des Betroffenen nach $\mathbb{1 1} \mathrm{Abs}$. 3 GenDG.

Die Gegenmeinung lehnt die uneingeschränkte Exzeptionalität genetischer Daten ab. Für Befunde von genetischen Untersuchungen wird deshalb die Ansicht vertreten, dass sie sich nicht von anderen Behandlungsdaten unterscheiden. ${ }^{193}$ Dies legt insbesondere auch ein Vergleich mit anderen prädiktiven Gesundheitsdaten nahe, die nicht durch genetische Untersuchungen gewonnen wurden. ${ }^{194}$ Denn für diese gilt das Gendiagnostikgesetz nicht, sodass es bei den allgemeinen datenschutzrechtlichen Grundsätzen bleibt. Dadurch kann dasselbe Untersuchungsergebnis, sofern es durch konventionelle prädiktive Untersuchungsmethoden ${ }^{195}$ (z.B. Stammbaumanalysen) und nicht durch eine genetische Untersuchung erzielt wurde, auch in anonymisierter Form an Dritte weitergegeben werden, da es nicht dem Anwendungsbereich des Gendiagnostikgesetzes unterfällt. Die anonymisierte Information, die an Dritte weitergegeben wird (das Vorliegen einer Erbkrankheit bei einer nicht identifizierbaren Person), ist in beiden Fällen, ob Befund einer genetischen oder konventionellen Untersuchung, dieselbe. Somit erscheint eine Unterscheidung der inhaltlich identischen Information gestützt lediglich auf die Unterschiedlichkeit der Diagnosemethode ${ }^{196}$ nicht gerechtfertigt.

Der zuletzt ausgeführten Ansicht ist zuzustimmen, sofern es sich bei den Ergebnissen nicht um individuelle DNS-Sequenzen oder nennenswerte Teile des Genoms handelt, sondern lediglich um isolierte genetische Befunde (z.B. das Vorliegen einer Chorea Huntington Mutation) als Interpretation einzelner DNS-Sequenzen. ${ }^{197}$ In diesen Fällen ist die Weitergabe des Befundergebnisses losgelöst vom „genetischen Fingerabdruck" einer individuellen Person in anonymisierter Form an Dritte nicht durch $\mathbb{s} 11$ Abs. 3 GenDG ausgeschlossen.

\section{Aufbewahrungsfristen und Vernichtungspflicht}

Nach $\mathbb{1} 12$ Abs. 1 S. 1 GenDG sind die Ergebnisse genetischer Untersuchungen und Analysen zehn Jahre in den Untersuchungsunterlagen aufzubewahren. Nach Ablauf

\footnotetext{
190 Vossenkuhl, Der Schutz genetischer Daten unter besonderer Berücksichtigung des Gendiagnostikgesetzes, S. 97

191 Vgl. Menzel, in: Sokol (Hg.), Der gläserne Mensch - DNA-Analysen, eine Herausforderung an den Datenschutz, S. 6.

192 Vossenkuhl, Der Schutz genetischer Daten unter besonderer Berücksichtigung des Gendiagnostikgesetzes, S. 97

193 Taupitz, in: Honnefelder/Propping (Hg.), Was wissen wir, wenn wir das menschliche Genom kennen?, S. $268 f$

194 Taupitz, in: Honnefelder/Propping (Hg.), Was wissen wir, wenn wir das menschliche Genom kennen?, S. 267f.

195 Vgl. Damm/König, MedR 2008, 62, 65f.

196 Vossenkuhl, Der Schutz genetischer Daten unter besonderer Berücksichtigung des Gendiagnostikgesetzes, S. 95

197 Zur Fragen der Anonymität in diesem Zusammenhang s.a. oben S. 19, Fn. 45.
} 
der Aufbewahrungsfrist sind die Daten in der Regel nach $\mathbb{1} 12$ Abs. 1 S. 2 Nr. 1 GenDG zu vernichten. Wenn Grund zur Annahme besteht, dass durch eine Vernichtung schutzwürdige Interessen der betroffenen Person beeinträchtigt würden oder wenn die betroffene Person eine längere Aufbewahrung schriftlich verlangt, sind die Daten zu sperren statt zu vernichten ( $\$ 12$ Abs. 1 S. 3 GenDG).

Dies entspricht im Wesentlichen der aus dem allgemeinen Datenschutzrecht bekannten Löschungsregelung (vgl. $\mathbb{3} 3$ Abs. 2, 3 BDSG). Für eine eher medizinrechtliche Regelung ist dies aber ungewöhnlich, da ansonsten im Medizinrecht zwar vielfach Aufbewahrungspflichten statuiert werden, ${ }^{198}$ sich eine Pflicht zur Löschung nach Ablauf der Aufbewahrungsfristen aber nur aus dem allgemeinen Datenschutzrecht ergibt. Vor diesem Hintergrund hebt die explizite Regelung in $\$ 12$ Abs. 1 S. 2 GenDG die besondere Sensibilität genetischer Daten hervor. ${ }^{199}$

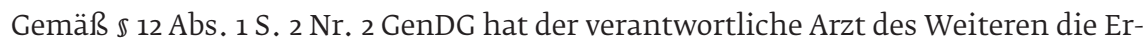
gebnisse genetischer Untersuchungen und Analysen unverzüglich in den Untersuchungsunterlagen über die betroffene Person zu vernichten, wenn die Person sich dazu entschieden hat, dass die Ergebnisse zu vernichten sind. Dies wird auch nochmals durch $\mathbb{1 2} \mathrm{Abs}$. 2 GenDG deutlich, wonach die Verpflichtung zur Aufbewahrung, Vernichtung und Sperrung des Ergebnisses nach $\mathbb{} 12$ Abs. 1 GenDG auch für die beauftragte Person oder Einrichtung gilt, die die genetischen Analysen durchführt.

Die Vernichtung muss nach den datenschutzrechtlichen Anforderungen an die Vernichtung sensibler Daten erfolgen. Es muss garantiert sein, dass die Ergebnisse der genetischen Untersuchung oder Analysen irreversibel vernichtet werden. ${ }^{200}$ Die im Ausnahmefall an die Stelle der Vernichtung tretende Sperrung der Ergebnisse entspricht $\$ 3$ Abs. 4 Nr. 4 BDSG und somit dem Kennzeichnen gespeicherter personenbezogener Daten, um ihre weitere Verarbeitung oder Nutzung einzuschränken. ${ }^{201}$ Die Frage, ob für solche gesperrte genetische Daten auch die Ausnahme des $\mathbb{s} 35$ Abs. 8 Nr. 1 BDSG Anwendung findet, wonach gesperrte Daten unter Umständen für wissenschaftliche Zwecke ohne Einwilligung des Betroffenen übermittelt oder genutzt werden dürfen, wird wegen der speziellen Eigenart genetischer Daten verneint. ${ }^{202}$

Somit muss die Vernichtungspflicht ohne gesonderte Einwilligung auch bei der Sekundärnutzung der genetischen Daten zu Forschungszwecken beachtet werden und durch das jeweilige System gewährleistet sein.

\subsubsection{Fazit}

Das Gendiagnostikgesetz findet bei der Sekundärnutzung im Behandlungszusammenhang gewonnener genetischer Daten zu Forschungszwecken Anwendung. Deshalb sind die spezialgesetzlichen Bestimmungen und Anforderungen des Cendiagnostikgesetzes bei der Sekundärnutzung zu beachten und umzusetzen.

198 Neben der Aufbewahrung aufgrund einer Rechtsvorschrift (wie $\S 28$ Abs. 3 Röntgenverordnung, $\S 14$ Abs. 3 TFG oder den $\S 10$ MBO-Ä entsprechende Regelungen der Landesärztekammern) dürfte eine solche für die Dauer der zivilrechtlichen Verjährung eventueller Haftungsansprüche wegen Behandlungsfehlern zulässig sein. Den zuletzt genannten Aspekt dürfte man unter die in \35 Abs. 2 S. 2 Nr. 3, Abs. 3 Nr. 1 BDSG ebenfalls erwähnten vertraglichen Aufbewahrungsfristen subsumieren können.

199 Schillhorn/Heidemann: GenDG, $\$ 12$ Rdnr. 6.

200 Schillhorn/Heidemann, GenDG, $\$ 12$ Rdnr. 16.

201 Fenger, in: Spickhoff (Hg.), Medizinrecht, GenDG, $\oint 12$ Rdnr. 2.

202 Kern, in Kern (Hg.), GenDG, $§ 12$ Rdnr. 9. 


\subsubsection{Transplantationsgesetz (TPG)}

Das Transplantationsgesetz findet gemäß $\mathbb{1}$ Abs. 2 TPG Anwendung auf Fälle der Spende und der Entnahme von menschlichen Organen oder Geweben zum Zweck der Übertragung sowie auf die eigentliche Übertragung der Organe oder der Gewebe einschließlich der Vorbereitung dieser Maßnahmen. Die Transplantation künstlicher Transplantate, z.B. von Hautersatzstoffen, sowie von Medizinprodukten im Sinne des $\mathbb{3} 3$ MPG sind vom Anwendungsbereich nicht umfasst. ${ }^{203}$ Weiterhin fallen nach $\mathbb{} 1$ Abs. 3 Nr. 1 TPG autologe Transplantationen, also Transplantationen, bei denen Gewebe, das innerhalb ein und desselben chirurgischen Eingriffs einer Person entnommen wird, um auf diese rückübertragen zu werden, nicht in den Anwendungsbereich des Transplantationsgesetzes. Außerdem gilt das Gesetz nach $\mathbb{1} 1$ Abs. 3 Nr. 2 TPG nicht für Blut und Blutbestandteile, wofür das Transfusionsgesetz (TFG) Anwendung findet. ${ }^{204}$ Um die Bereitschaft zur Organspende in Deutschland zu fördern, so das Gesetzesziel nach $\mathbb{1}$ Abs. 1 TPG, ist es unter anderem Aufgabe dieses Gesetzes, die Anonymität des Organ- bzw. Gewebespenders weitestgehend zu garantieren.

\subsubsection{Grundsatz der Zweckgebundenheit}

Als Sonderregelung zu $\mathbb{s} 4$, 4a BDSG regelt $\mathbb{7}$ Abs. 1 TPG die Zulässigkeit der Datenerhebung für bestimmte Zwecke. ${ }^{205}$ Demnach ist die Erhebung und Verwendung personenbezogener Daten eines möglichen Organ- oder Gewebespenders, eines Angehörigen oder einer nahestehenden Person und die Übermittlung dieser Daten an die nach $\mathbb{7}$ Abs. 3 S. 1 TPG auskunftsberechtigten Personen zulässig, soweit dies erforderlich ist

1. zur Klärung, ob eine Organ- oder Gewebeentnahme nach $\mathbb{3} 3$ Abs. 1 und 2, $\mathbb{4} 4$ Abs. 1 bis 3 sowie $\sqrt{9}$ Abs. 3 S. 2 TPG zulässig ist und ob ihr medizinische Gründe entgegenstehen,

2. zur Unterrichtung der nächsten Angehörigen nach $₫ 3$ Abs. 3 S. 1 TPG,

3. zur Organ- und Spendercharakterisierung nach $\mathbb{1}$ 10a TPG,

4. zur Rückverfolgung nach $\$ 13$ Abs. 1 TPG oder

5. zur Meldung schwerwiegender Zwischenfälle und schwerwiegender unerwünschter Reaktionen auf der Grundlage der Rechtsverordnung nach $\mathbb{S} 13$ Abs. 4 TPG.

Grundsätzlich ist die Erhebung und Verwendung personenbezogener Daten im Rahmen des TPG somit zweckgebunden. Dies stellt auch $\mathbb{1} 14$ Abs. 2 S. 3 TPG klar, der vorschreibt, dass die im Rahmen dieses Gesetzes erhobenen personenbezogenen Daten nicht für andere als in diesem Gesetz genannte Zwecke verwendet werden dürfen. Nach $\mathbb{1} 14$ Abs. 2 S. 1 und S. 2 TPG dürfen personenbezogene Daten der Spender und der Empfänger nicht offenbart werden. Ein Verstoß hiergegen ist nach $\mathbb{} 19$ Abs. 3 TPG strafbar.

203 Walter, in: Spickhoff (Hg.), Medizinrecht, TPG, $\ 1$ Rdnr. 1.

204 Zum TFG siehe unten S. 62 .

205 Walter, in: Spickhoff (Hg.), Medizinrecht, TPG, § 7 Rdnr. 1. 


\subsubsection{Forschungsklausel}

$\mathbb{\$} 14$ Abs. 2a TPG macht jedoch vom Grundsatz der Zweckgebundenheit der Daten eine Ausnahme. Demnach dürfen Ärzte und anderes wissenschaftliches Personal des Entnahmekrankenhauses, des Transplantationszentrums, der Koordinierungsstelle

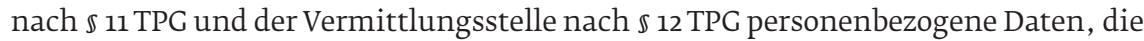
im Rahmen der Organ- und Spendercharakterisierung beim Organ- oder Gewebespender oder im Rahmen der Organ- oder Gewebeübertragung beim Organ- oder Gewebeempfänger erhoben worden sind, abweichend von $\mathbb{1} 14 \mathrm{Abs}$. 2 S. 3 TPG für eigene wissenschaftliche Forschungsvorhaben verwenden. Diese Daten dürfen für ein bestimmtes Forschungsvorhaben an Dritte und andere als die in S. 1 genannten Personen übermittelt und von diesen verwendet werden, wenn 1. die Daten der betroffenen Person nicht mehr zugeordnet werden können, 2. im Falle, dass der Forschungszweck die Möglichkeit der Zuordnung erfordert, die betroffene Person eingewilligt hat oder 3. im Falle, dass weder auf die Zuordnungsmöglichkeit verzichtet noch die Einwilligung mit verhältnismäßigem Aufwand eingeholt werden kann, das öffentliche Interesse an der Durchführung des Forschungsvorhabens die schützenswerten Interessen der betroffenen Person überwiegt und der Forschungszweck nicht auf andere Weise zu erreichen ist.

Die personenbezogenen Daten sind, soweit dies nach dem Forschungszweck möglich ist und keinen im Verhältnis zu dem angestrebten Schutzzweck unverhältnismäßigen Aufwand erfordert, zu anonymisieren oder, solange eine Anonymisierung noch nicht möglich ist, zu pseudonymisieren ( $\$ 14$ Abs. 2a S. 3 TPG).

\subsubsection{Fazit}

Unter den Voraussetzungen des $\mathbb{} 14$ Abs. 2a TPG ist die Sekundärnutzung von personenbezogenen Daten zu Forschungszwecken im Anwendungsbereich des TPG zulässig. ${ }^{206}$

\subsubsection{Medizinproduktegesetz (MPG)}

Das Medizinproduktegesetz hat nach $\mathbb{\$} 1$ MPG das Ziel, den Verkehr mit Medizinprodukten zu regeln und dadurch für die Sicherheit, Eignung und Leistung der Medizinprodukte sowie die Gesundheit und den erforderlichen Schutz der Patienten, Anwender und Dritter zu sorgen. Der weitgefasste Begriff Medizinprodukte beschreibt nach $\$ 3$ Nr. 1 MPG grundsätzlich Instrumente, Apparate, Vorrichtungen, Software, aber auch Stoffe und Zubereitungen aus Stoffen zur Anwendung für Menschen, die zu diagnostischen oder therapeutischen Zwecken eingesetzt werden.

\subsubsection{Verhältnis von MPG und allgemeinem Datenschutzrecht}

$\mathbb{} 2$ Abs. 4 MPG regelt die parallele Anwendbarkeit anderer Rechtsvorschriften neben dem MPG. Demnach bleiben die Rechtsvorschriften über Geheimhaltung und Datenschutz von den Vorschriften des MPG unberührt. Durch diese explizite Regelung soll 
„verdeutlicht werden, dass das Vertraulichkeitsgebot des Bundesdatenschutzgesetzes und der Landesdatenschutzgesetze im privaten und im behördlichen Umgang mit entsprechend personenbezogenen Daten nicht durch die Regelungen im MPG (hier insbesondere im fünften Abschnitt, Überwachung und Schutz vor Risiken) ausgeschlossen werden sollte“. ${ }^{207}$ Trotz dieses Grundsatzes enthält das MPG vereinzelt Bestimmungen, die auch eine personenbezogene Datenverarbeitung erlauben:

So regelt $\$ 29$ MPG die zentrale Erfassung und Bewertung von Risiken bei Medizinprodukten, welche als Sonderfall der einrichtungsübergreifenden Qualitätssicherung angesehen werden kann. $\mathbb{2} 29$ Abs. 2 MPG stellt hierbei besondere Anforderungen an

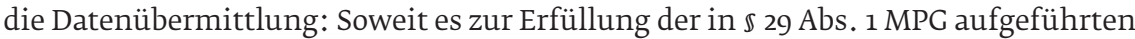
Aufgaben erforderlich ist, dürfen an die danach zuständigen Behörden auch Name, Anschrift und Geburtsdatum von Patienten, Anwendern oder Dritten übermittelt werden ( $\$ 29$ Abs. 2 S. 1 MPG). Die nach $\$ 29$ Abs. 1 MPG zuständige Behörde darf die nach Landesrecht zuständige Behörde auf Ersuchen über die von ihr gemeldeten Fälle und die festgestellten Erkenntnisse in Bezug auf personenbezogene Daten unterrichten (\$29 Abs. 2 S. 2 MPG).

Bei der Zusammenarbeit zwischen Bundesinstitut für Arzneimittel und Medizinprodukte bzw. des Paul-Ehrlich-Instituts und den in $\$ 29$ Abs. 3 MPG genannten anderen Stellen dürfen jedoch keine personenbezogenen Daten von Patienten übermittelt werden, $\mathbb{5} 29$ Abs. 2 S. 3 MPG. Die Übermittlung von personenbezogenen Daten ist hierbei in jedem Fall unzulässig. ${ }^{208}$

\subsubsection{Fazit}

Der Umgang mit personenbezogenen Daten ist im Rahmen des MPG somit lediglich zum Zwecke der zentralen Erfassung und Bewertung von Risiken bei Medizinprodukten geregelt, welche nur unter Beachtung der recht engen Restriktionen des $\$ 29$ MPC zulässig ist. Im Übrigen, also für sonstige Maßnahmen der Qualitätssicherung oder die Forschung zu Medizinprodukten, bleibt es bei der Anwendung der jeweils gültigen allgemeinen datenschutzrechtlichen Normen, die nach Sitz und Art der verantwortlichen Stelle zu bestimmen sind. ${ }^{209}$

\subsubsection{Transfusionsgesetz (TFG)}

Das Transfusionsgesetz regelt die Gewinnung und den Umgang mit Blut und Blutbestandteilen, insbesondere also den Bereich der Blutspende und Spenderimmunisierung. Zweck des Transfusionsgesetzes ist es nach $\mathbb{1} 1$ TFG insbesondere, für eine sichere Gewinnung von Blut und Blutbestandteilen und für eine gesicherte und sichere Versorgung der Bevölkerung mit Blutprodukten zu sorgen und die Selbstversorgung mit Blut und Plasma auf der Basis der freiwilligen und unentgeltlichen Blutspende zu fördern. Auch die im Rahmen von Blutspenden oder Spenderimmunisierungen gesammelten Daten können für eine Sekundärnutzung von Interesse sein.

207 Lücker, in: Spickhoff (Hg.), Medizinrecht, MPG, \$ 2 Rdnr. 9.

208 Lücker, in: Spickhoff (Hg.), Medizinrecht, MPG, \ 29 Rdnr. 3.

209 S. dazu unten Kap. I.6, S. $75 f f$. 


\subsubsection{Modifizierung des allgemeinen Datenschutzrechts}

Nach $\mathbb{1} 11$ Abs. 2 TFG dürfen die Spendeeinrichtungen personenbezogene Daten der spendewilligen und spendenden Personen im Rahmen der Gewinnung von Blut und Blutbestandteilen erheben, verarbeiten und nutzen, soweit das für die in $\mathbb{1 1} \mathrm{Abs} .1$ TFG genannten Zwecke erforderlich ist. Als erforderliche Zwecke nennt $\$ 11$ Abs. 1 S . 1 TFG neben dem Zweck der Erfüllung der ärztlichen Dokumentationspflichten auch die im Transfusionsgesetz geregelten Zwecke, die Dokumentation für Zwecke der ärztlichen Behandlung der spendenden Person und für Zwecke der Risikoerfassung nach dem Arzneimittelgesetz (vgl. $\mathbb{s} 62 \mathrm{ff}$. AMG).

Für die Aufzeichnungen nach $\mathbb{1} 11$ Abs. 1 S. 1 TFG gelten spezielle Aufbewahrungspflichten. Im Regelfall sind die Aufzeichnungen mindestens 15 Jahre, im Fall der Hyperimmunisierung nach $\$ \$ 8$ und 9 Abs. 1 TFC mindestens 20 Jahre aufzubewahren. Für Angaben, die für die Rückverfolgung benötigt werden, gilt eine Aufbewahrungspflicht von mindestens 30 Jahren ( $\$ 11$ Abs. 1 S. 2 TFG). Die Aufbewahrungsfristen stehen unter der Bedingung, dass die Aufbewahrung erforderlich sein muss. Ist dies nicht mehr der Fall, sind die Aufzeichnungen zu vernichten oder zu löschen. Dies ist beispielsweise nach dem Tod des Patienten der Fall, falls die Akten dann nicht mehr benötigt werden, jedoch kann es aus wissenschaftlichen Gründen auch bei der normalen Aufbewahrungsfrist bleiben. ${ }^{210}$ Werden die Aufzeichnungen länger als dreißig Jahre nach der letzten bei der Spendeeinrichtung dokumentierten Spende desselben Spenders aufbewahrt, sind sie zu anonymisieren, $\mathbb{1} 11$ Abs. 1 S. 4 TFG. Die Dokumentation der Aufzeichnungen muss gemäß $\mathbb{s} 11$ Abs. 1 S. 3 TFG so geordnet sein, dass ein unverzüglicher Zugriff möglich ist, womit hier ein sofortiger Zugriff 211 gemeint ist.

Die Spendeeinrichtungen nach $\$ 11$ Abs. 2 TFG übermitteln die protokollierten Daten den zuständigen Behörden und der zuständigen Bundesoberbehörde (das Paul-Ehr-

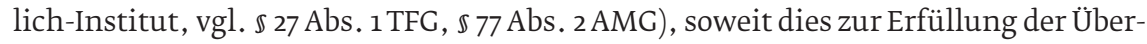
wachungsaufgaben nach dem Arzneimittelgesetz oder zur Verfolgung von Straftaten oder Ordnungswidrigkeiten, die im engen Zusammenhang mit der Spendeentnahme stehen, erforderlich ist. Zur Risikoerfassung nach dem Arzneimittelgesetz sind das Geburtsdatum und das Geschlecht der zu behandelnden Person anzugeben, $\mathbb{1} 11 \mathrm{Abs} .2$ S. 3 TFG.

Eine dem $₫ 11$ TFG vergleichbare Regelung findet sich in $\mathbb{1} 14$ TFG, der die Dokumentation bei der Anwendung von Blutprodukten und von gentechnisch hergestellten Plasmaproteinen zur Behandlung von Hämostasestörungen regelt.

Auch im Rahmen von $\$ 14$ TFG besteht die ärztliche Dokumentationspflicht für die im Transfusionsgesetz geregelten Zwecke, für Zwecke der ärztlichen Behandlung der von der Anwendung betroffenen Personen und für Zwecke der Risikoerfassung nach dem Arzneimittelgesetz. Zusätzlich schreibt $\$ 14$ Abs. 1 S. 2 TFG vor, dass die Dokumentation die Aufklärung und die Einwilligungserklärungen, das Ergebnis der Blutgruppenbestimmung, soweit die Blutprodukte blutgruppenspezifisch angewendet werden, die durchgeführten Untersuchungen sowie die Darstellung von Wirkungen und unerwünschten Ereignissen zu umfassen hat.

210 Deutsch, in: Spickhoff (Hg.), Medizinrecht, TFG, $\$ 11$ Rdnr. 3.

211 Deutsch, in: Spickhoff (Hg.), Medizinrecht, TFG, § 11 Rdnr. 4. 
Für die Aufbewahrungspflicht schreibt $\$ 14$ Abs. 3 TFG vor, dass die Aufzeichnungen, einschließlich der EDV-erfassten Daten, mindestens 15 Jahre lang aufbewahrt werden müssen. Für die Daten nach $\$ 14$ Abs. 2 TFG (Patientenidentifikationsnummer, Chargenbezeichnung, Pharmazentralnummer, Datum und Uhrzeit der Anwendung) ist eine Aufbewahrungsflicht von mindestens 30 Jahren vorgeschrieben. Wie auch in $\mathbb{S} 11$ Abs. 1 TFG steht die Aufbewahrungspflicht unter der Bedingung, dass die Aufbewahrung weiterhin erforderlich ist und dass die Daten andernfalls zu vernichten oder zu löschen sind. Nach $\mathbb{1} 14$ Abs. 3 S. 3 TFG müssen die Aufzeichnungen zu Zwecken der Rückverfolgung unverzüglich (sofort ${ }^{212}$ ) verfügbar sein. Werden die Aufzeichnungen länger als dreißig Jahre aufbewahrt, sind sie zu anonymisieren, $\mathbb{S} 14 \mathrm{Abs} .3$ S. 4 TFG.

Gemäß $\mathbb{1} 14$ Abs. 4 TFG dürfen die Einrichtungen der Krankenversorgung personenbezogene Daten der zu behandelnden Personen erheben, verarbeiten und nutzen, soweit das für die in $\mathbb{1} 14$ Abs. 1 TFG genannten Zwecke erforderlich ist. Außerdem übermitteln sie nach $\mathbb{1} 14$ Abs. 4 S. 2 TFG die dokumentierten Daten den zuständigen Behörden, soweit dies zur Verfolgung von Straftaten, die im engen Zusammenhang mit der Anwendung von Blutprodukten stehen, erforderlich ist. Zur Risikoerfassung nach dem Arzneimittelgesetz sind das Geburtsdatum und das Geschlecht der zu behandelnden Person anzugeben, $\mathbb{5} 14$ Abs. 4 S. 3 TFG.

Sowohl $\mathbb{1} 11$ Abs. 2 als auch $\mathbb{s} 14$ Abs. 4 TFG stellen somit darauf ab, dass das Erheben, Verarbeiten und Nutzen personenbezogener Daten durch die Spendeeinrichtungen bzw. die Einrichtungen der Krankenversorge zulässig ist, soweit es im Rahmen der Dokumentationspflicht zu den in $\mathbb{1} 11$ Abs. 1 und $\mathbb{1} 14$ Abs. 2 TFG genannten Zwecken erforderlich ist. Auch die Übermittlung an die zuständigen Behörden und die Bundesoberbehörde ( $\$ 27 \mathrm{Abs}$. 1 TFG) ist zweckbestimmt und dient deren Überwachungsaufgaben nach dem AMG ( $\$ \$ S 2 \mathrm{ff}$. AMG).

\subsubsection{Fazit}

Datenschutzrechtliche Anforderungen werden durch diese Normen modifiziert, um die Rückverfolgung zum Spender für die im TFG genannten Zwecke zu ermöglichen. ${ }^{213}$ Bei diesen Zwecken handelt es sich aber lediglich um solche, die der Sicherheit der Spender und Empfänger dienen, was dem primären Behandlungszweck entspricht. Ergänzt wird dieser Zweck durch besondere Vorschriften zur Qualitätssicherung, insbesondere die Risikoerfassung nach dem Arzneimittelgesetz.

Die Sekundärnutzung der angefallenen Daten für sonstige Formen der Qualitätssicherung oder die Forschung ist im TFG dagegen nicht geregelt. Insoweit kann auf die im Übrigen jeweils anwendbaren Vorschriften wie das BDSC oder die Landeskrankenhausgesetze zurückgegriffen werden.

\subsubsection{Arzneimittelgesetz (AMG)}

Zweck des Arzneimittelgesetzes ist es nach $\$ 1$ AMG, im Interesse einer ordnungsgemäßen Arzneimittelversorgung von Mensch und Tier für die Sicherheit im Verkehr

212 Deutsch, in: Spickhoff (Hg.), Medizinrecht, TFG, $§ 14$ Rdnr. 4.

213 Vgl. Deutsch, in: Spickhoff (Hg.), Medizinrecht, TFG, § 11 Rdnr. 1. 
mit Arzneimitteln, insbesondere für die Qualität, Wirksamkeit und Unbedenklichkeit der Arzneimittel, zu sorgen. Zu diesem Zwecke regelt das Arzneimittelgesetz unter anderem die Anforderungen an Arzneimittel, die Herstellung von Arzneimitteln sowie die Zulassung von Arzneimitteln und den Schutz des Menschen bei klinischen Prüfungen. Insbesondere die im Rahmen von klinischen Studien gewonnenen Daten könnten für eine Sekundärnutzung interessant sein.

In Bezug auf die Sekundärnutzung von Behandlungsdaten stellt sich zum einen die Frage, ob die im Rahmen von klinischen Studien für primäre Forschungszwecke und daneben möglicherweise auch für die Behandlung (bei kranken Probanden) gewonnenen Daten auch für sekundäre Forschungszwecke und solche der Qualitätssicherung verwendet werden dürfen. Zum anderen ist fraglich, ob und ggf. unter welchen Voraussetzungen die Vorschriften des AMG für die Sekundärnutzung von Arzneimitteltherapiedaten unabhängig von klinischen Studien Anwendung finden.

\subsubsection{Klinische Prüfung und nichtinterventionelle Prüfung}

Entscheidend für die Anwendung der Vorschriften des Arzneimittelgesetzes ist die Frage, ob es sich bei einer Studie um eine klinische Prüfung handelt.

\section{Klinische Prüfung}

$\mathbb{\$} 4$ Abs. 23 AMC definiert den für das Arzneimittelgesetz zentralen Begriff der klinischen Prüfung bei Menschen. Dies ist jede am Menschen durchgeführte Untersuchung, die dazu bestimmt ist, klinische oder pharmakologische Wirkungen von Arzneimitteln zu erforschen oder nachzuweisen oder Nebenwirkungen festzustellen oder die Resorption, die Verteilung, den Stoffwechsel oder die Ausscheidung zu untersuchen, mit dem Ziel, sich von der Unbedenklichkeit oder Wirksamkeit der Arzneimittel zu überzeugen.

Die klinische Prüfung muss nicht zwangsläufig in einer Klinik durchgeführt werden. Sie kann auch bei einem niedergelassenen Arzt erfolgen, wenn Zweck der Prüfung ist, über die Behandlung des Einzelfalles hinaus wissenschaftliche Erkenntnisse in Bezug auf die in $\mathbb{4} 4$ Abs. 23 AMG genannten Eigenschaften des Arzneimittels zu gewinnen. ${ }^{214}$

Auf solche klinischen Prüfungen finden vor allem zum Schutz der Probanden die $\mathbb{S} \mathbb{S} 4$ off. des AMG Anwendung, welche unter anderem Sonderregeln auch zum Datenschutz enthalten. ${ }^{215}$

\section{Nichtinterventionelle Prüfung}

Keine klinischen Prüfungen im eben ausgeführten Sinn sind jedoch nichtinterventionelle Untersuchungen ( $\$ 4$ Abs. 23 S. 2 AMG). Solche nichtinterventionellen Prüfungen sind Untersuchungen, in deren Rahmen Daten aus der Beobachtung des Verlaufs einer Standardtherapie ohne studienspezifische Intervention analysiert werden. So sollen Erkenntnisse aus der Behandlung von Personen mit Arzneimitteln anhand epidemiologischer Methoden gewonnen werden; dabei folgt die Behandlung ein-

214 Rehmann, in: Rehmann, AMG, Vorbemerkung zu $\$ 40-42 a$ Rdnr. 3.

215 Näheres siehe unten $S$. $68 f$. 
schließlich der Diagnose und Überwachung nicht einem vorab festgelegten Prüfplan, sondern ausschließlich der ärztlichen Praxis; soweit ein zulassungs- oder genehmigungspflichtiges Arzneimittel eingesetzt wird, erfolgt dies ferner gemäß den in der Zulassung oder der Genehmigung festgelegten Angaben für seine Anwendung ( $\$ 23$ Abs. 23 S. 3 AMG). Im Rahmen von nichtinterventionellen Studien werden folglich nur Daten analysiert und kein unmittelbarer Einfluss auf die Therapie genommen.

Auf solche nichtinterventionellen Prüfungen finden die Vorschriften des AMG weitgehend keine Anwendung. Insbesondere gelten die $\$ \mathbb{\$}$ 4off. AMG nicht. ${ }^{216}$ Die Notwendigkeit einer Aufklärung und Einwilligung des Patienten für die Teilnahme an solchen Studien sowie gegebenenfalls die Anforderungen an solche Erklärungen richten sich damit nicht nach dem AMG, ${ }^{217}$ sondern nach den jeweils anwendbaren allgemeinen Vorschriften (wie dem BDSG oder den LKHG).

Typischer Fall von nichtinterventionellen Studien sind Anwendungsbeobachtungen nach $\$ 67$ Abs. 6 AMC. ${ }^{218} \mathbb{\$} 67$ Abs. 6 S. 1 AMG schreibt lediglich eine Anzeigepflicht für denjenigen vor, der Anwendungsbeobachtungen durchführt. Welche Rahmenbedingungen für die Durchführung von Anwendungsbeobachtungen (wie etwa eine datenschutzrechtliche Aufklärung des Patienten) einzuhalten sind, ist dagegen im AMG nicht geregelt. ${ }^{219}$ Bei einer vollständigen Anonymisierung der im Rahmen einer Anwendungsbeobachtung erhobenen Patienten- bzw. Gesundheitsdaten ist die Einwilligung des Patienten nicht notwendig. ${ }^{220}$

Die Sekundärnutzung von Behandlungsdaten aus nichtinterventionellen Studien bzw. im Rahmen solcher Studien wird somit nicht durch das Arzneimittelgesetz eingeschränkt.

\section{Einordnung von Phase-IV-Studien}

Einen Sonderfall stellen sogenannte Phase-IV-Studien dar. Dies sind Studien mit Arzneimitteln nach deren Zulassung. Sie dienen der weiteren Bewertung des Verhältnisses von Risiko und Nutzen in der breiten Anwendungspraxis, also an größeren Patientengruppen, und/oder an speziellen Patientenkollektiven.

In Bezug auf die eigentliche Arzneimitteltherapie sind diese Studien zwar nichtinterventionell, denn es wird lediglich ein bereits zugelassenes Medikament nach Maßgabe seiner Zulassung verabreicht. ${ }^{221}$ Im Unterschied zu bloßen Anwendungsbeobachtungen werden allerdings bei Phase-IV-Studien in aller Regel Diagnose- bzw. Überwachungsverfahren eingesetzt, die über das Maß der normalen ärztlichen Praxis hinausgehen. ${ }^{222}$ Insoweit kann von einer diagnostischen Intervention gesprochen werden, weshalb Phase-IV-Studien als klinische Prüfungen angesehen werden, auf die das AMG vollständig Anwendung findet. Auch hierbei zu erhebende Daten und deren Nutzung unterliegen daher den Vorschriften des Arzneimittelgesetzes.

216 Zum Sonderfall der Phase-IV-Studien siehe sogleich S. 66.

217 Vgl. Sander, AMG, $\$ 40$ Rdnr. 4b.

218 Wachenhausen, in: Kügel/Müller/Hofmann (Hg.): AMG, \$ 4 Rdnr. 160.

219 Wachenhausen, in: Kügel/Müller/Hofmann (Hg.), AMG, \ 4 Rdnr. $161 f$. 220 Wachenhausen, in: Kügel/Müller/Hofmann (Hg.), AMG, $\$ 4$ Rdnr. 162. Anders im Fall der bloßen Pseudonymisierung, beispiels-
weise zur Nachverfolgung der Probanden/Patienten über einen längeren Zeitraum in Longitudinalstudien, wo sich durchaus ein Einwilligungserfordernis ergeben kann, soweit nicht gesetzliche Forschungserlaubnisse greifen.

221 Vgl. Listl, in: Spickhoff (Hg.), Medizinrecht, AMG, \& 40 Rdnr. 4.

222 Diener/Klümper, PharmR 2010, 433, 434 m.w.N. 


\subsubsection{Keine Anwendung auf reine Heilversuche}

Auch Heilversuche sind von klinischen Prüfungen abzugrenzen. Gegenstand des Heilversuches ist es, bei der individuellen Behandlung eines Patienten, bei dem eine herkömmliche Standardbehandlung keine Wirkung zeigt, neuartige therapeutische oder diagnostische Maßnahmen anzuwenden. ${ }^{223}$ Denklogisch können Heilversuche nur dann vorliegen, wenn es sich um einen kranken Patienten handelt, da sonst nicht die Heilung der Zweck sein kann. ${ }^{22}$ Bei einem Heilversuch wird somit mit einer Therapie primär ein konkreter Therapieerfolg und nicht wie bei einer klinischen Studie wissenschaftlicher Erkenntnisgewinn angestrebt. Da es sich bei einem reinen Heilversuch nicht um eine klinische Studie handelt, und um Patienten den Zugang zum konkreten Heilversuch nicht zu erschweren, finden die $\mathbb{s} 4$ off. AMG keine Anwendung. ${ }^{225}$

Die Abgrenzung zwischen reinem Heilversuch und klinischer Studie ist jedoch nicht in jedem Fall einfach. ${ }^{226}$ Abgrenzungskriterium ist dabei allein der Zweck der durchgeführten Behandlung ${ }^{227}$ und nicht etwa die Anzahl der Patienten, an denen der Heilversuch unternommen wird. ${ }^{228}$

Werden wissenschaftlicher Erkenntnisgewinn und Heilversuch kombiniert, soll also bei einer volljährigen Person, die an einer Krankheit leidet, zu deren Behandlung das zu prüfende Arzneimittel angewendet werden soll, eine klinische Prüfung durchgeführt werden (sogenannter therapeutischer Versuch), findet $\$ 41 \mathrm{AMC}$ zusätzlich zu $\mathbb{} 40$ AMG Anwendung.

Die Nichtanwendbarkeit des $\mathbb{S}$ 4off. AMG gilt somit nur für reine Heilversuche.

\subsubsection{Keine Anwendung auf Arzneimitteltherapiedaten bei Standardbehandlungen bzw. Pharmakovigilanzdaten}

Da die $₫ \mathbb{S}$ 4off. AMG grundsätzlich nur bei klinischen Prüfungen und nicht bei Heilversuchen Anwendung finden, sind folglich erst recht auch Arzneimitteldaten, die im Rahmen von Standardbehandlungen anfallen, von der Anwendung der $\$ \mathbb{\$} 4$ off. AMG ausgenommen. Standardbehandlungen sind in Abgrenzung von Heilversuchen ${ }^{229}$ Behandlungen mit etablierten Therapiemethoden und zugelassenen Arzneimitteln innerhalb ihres Indikationsgebiets. Für solche „normalen“ Behandlungen finden die $\mathbb{S}$ 4off. AMG keine Anwendung.

Auch für Arzneimitteltherapiedaten aus dem Bereich der Pharmakovigilanz kommt es durch das Arzneimittelgesetz zu keiner Modifizierung in Bezug auf die Sekundärnutzung der Behandlungsdaten.

Der Begriff Pharmakovigilanz bezeichnet die Nachmarktkontrolle von Arzneimitteln in Form der Dauerüberwachung zur Gewährleistung der Arzneimittelsicherheit als Ergänzung des Zulassungsverfahrens. ${ }^{230}$ Eine solche Beobachtung von Arzneimitteln nach deren Markteinführung ist insbesondere deshalb geboten, da trotz der Unbe-

223 Helle/Frölich/Haindl, NJW 2002, 857, 860.

224 Bender, MedR 2005, 511, 512.

225 Listl, in: Spickhoff (Hg.): Medizinrecht, AMG, \40 Rdnr. 5.

226 Helle/Frölich/Haindl, NJW 2002, 857, 860.

227 Rehmann, in Rehmann: AMG, Vorbemerkung zu $§ 40-42 a$ Rdnr. 3.

228 Bender, MedR 2005, 511, 512.

229 Helle/Frölich/Haindl, NJW 2002, 857, 860.

230 Schickert, in: Kügel/Müller/Hofmann (Hg.), AMG, Vorbemerkungen zu $§ 62$ Rdnr. 1. 
denklichkeitsprüfung im Zulassungs- und Registrierungsverfahren nicht schon sämtliche Risiken des Arzneimittels (z.B. Nebenwirkungen, Wechselwirkungen mit anderen Arzneimitteln oder unerwünschte Wirkungen bei länger andauernder Einnahme, vgl. $\mathbb{} 4$ Abs. 13 und Abs. 27 AMG) festgestellt werden können. ${ }^{231} \mathrm{Zu}$ diesem Zwecke sieht das Arzneimittelgesetz ein System für die Überwachung von Arzneimittelrisiken vor ( $\$ \$ 52$ bis $63 j \mathrm{AMG}$ ).

Nach $\mathbb{s}$ 63bff. AMG treffen den Inhaber der Zulassung des Arzneimittels vielfältige Dokumentations- und Meldepflichten von Arzneimittelrisiken. Die $\$ \$$ 62ff. AMG verpflichten jedoch nur den Inhaber der Zulassung zur Dokumentation und Meldung von Arzneimittelrisiken. Für Ärzte gibt es keine gesetzliche Pflicht, Arzneimittelrisiken zu melden. Allerdings ergibt sich für sie eine solche Pflicht aus den jeweiligen Berufsordnungen (vgl. $\$ 6 \mathrm{MBO}-\ddot{A}) .{ }^{232}$

Für Arzneimitteltherapiedaten, die im Rahmen einer ärztlichen Behandlung außerhalb einer klinischen Studie anfallen, werden somit die allgemeinen datenschutzrechtlichen Vorgaben in Bezug auf Behandlungsdaten nicht modifiziert. Insbesondere ergeben sich aus den $\$ \$ \$ ~ 62$ bis 63 j AMG keine speziellen datenschutzrechtlichen Vorgaben. ${ }^{233}$

\subsubsection{Datenschutzrechtliche Einwilligung bei klinischer Prüfung}

Für den Fall der klinischen Prüfung sind die $\$ \mathbb{\$} 40 \mathrm{off}$. AMG zu beachten. Nach $\mathbb{} 40$ Abs. 1 Nr. 3 Buchstabe c AMG darf eine klinische Prüfung eines Arzneimittels bei Menschen nur durchgeführt werden, wenn und solange die betroffene Person nach $\mathbb{S} 40$ Abs. 2a S. 1 und 2 AMG informiert worden ist und schriftlich eingewilligt hat. Die Einwilligung muss sich dabei ausdrücklich auch auf die Erhebung und Verarbeitung von Angaben über die Gesundheit beziehen. Eine gesonderte datenschutzrechtliche Einwilligung der betroffenen Person erscheint im Kontext der klinischen Prüfung von Arzneimitteln deshalb geboten, da sensible Gesundheitsdaten an Dritte (Sponsor, Behörden) notwendigerweise weitergegeben werden müssen. ${ }^{234}$ Die Informationspflicht nach $\mathbb{4} 4 \mathrm{O}$ Abs. 2a S. I AMG beinhaltet eine umfangreiche Information der betroffenen Person über Zweck und Umfang der Erhebung und Verwendung personenbezogener Daten, insbesondere von Gesundheitsdaten. Gemäß $\$ 40$ Abs. 2a S. 2 AMG ist die betroffene Person insbesondere darüber zu informieren, dass (1) die erhobenen Daten soweit erforderlich (a) zur Einsichtnahme durch die Überwachungsbehörde oder Beauftragte des Sponsors zur Überprüfung der ordnungsgemäßen Durchführung der klinischen Prüfung bereitgehalten werden, (b) pseudonymisiert an den Sponsor oder eine von diesem beauftragte Stelle zum Zwecke der wissenschaftlichen Auswertung weitergegeben werden, (c) im Falle eines Antrags auf Zulassung pseudonymisiert an den Antragsteller und die für die Zulassung zuständige Behörde weitergegeben werden, (d) im Falle unerwünschter Ereignisse des zu prüfenden Arzneimittels pseudonymisiert an den Sponsor und die zuständige Bundesoberbehörde sowie von dieser an die Europäische Datenbank weitergegeben werden, (2) die Einwilligung in den Datenumgang (nach Abs. 1 S. 3 Nr. 3 Buchstabe c) unwiderruflich ist, (3) im Falle eines Widerrufs der (nach Abs. 1 S. 3 Nr. 3 Buchstabe b) erklärten Ein-

231 Heßhaus, in: Spickhoff (Hg.), Medizinrecht, AMG, \$ 62 Rdnr. 1.

232 Schickert, in: Kügel/Müller/Hofmann (Hg.), AMG, Vorbemerkungen zu $\$ 62$ Rdnr. 5.

233 Meldungen im Rahmen der Pharmakovigilanz haben damit in Bezug auf die Patienten grundsätzlich anonym zu erfolgen, soweit nicht deren Einwilligung vorliegt.

234 Wachenhausen, in: Kügel/Müller/Hofmann (Hg.), AMG, \ 40 Rdnr. 85. 
willigung in die Studienteilnahme die gespeicherten Daten weiterhin verwendet werden dürfen, soweit dies erforderlich ist, um (a) Wirkungen des zu prüfenden Arzneimittels festzustellen, (b) sicherzustellen, dass schutzwürdige Interessen der betroffenen Person nicht beeinträchtigt werden, (c) der Pflicht zur Vorlage vollständiger Zulassungsunterlagen zu genügen, (4) die Daten bei den genannten Stellen für die auf Grund des $\mathbb{4} 42$ Abs. 3 AMG bestimmten Fristen gespeichert werden.

Widerruft der Betroffene seine Einwilligung nach $\mathbb{} 40$ Abs. 1 S. 3 Nr. 3 Buchstabe b AMG, haben die verantwortlichen Stellen unverzüglich zu prüfen, inwieweit die gespeicherten Daten für die in $\mathbb{} 40$ Abs. 2a S. 2 Nr. 3 AMG genannten Zwecke noch erforderlich sein können. Nicht mehr benötigte Daten sind unverzüglich zu löschen. Im Übrigen sind die erhobenen personenbezogenen Daten nach Ablauf der auf Grund des $\$ 42 \mathrm{Abs}$. 3 AMG bestimmten Fristen zu löschen, soweit nicht weitere gesetzliche, satzungsmäßige oder vertragliche Aufbewahrungsfristen entgegenstehen.

\subsubsection{Datenschutzrechtliche Vorgaben bei der Veröffentlichung der Ergebnisse klinischer Studien}

$\mathbb{S} 42 \mathrm{~b}$ Abs. 3 S. 4 AMG regelt, dass bei der Veröffentlichung der Ergebnisse klinischer Prüfungen mit Ausnahme des Namens und der Anschrift des pharmazeutischen Unternehmers oder des Sponsors sowie der Angabe des Namens und der Anschrift von nach $₫ 4$ a BDSG einwilligenden Prüfärzten die Berichte des pharmazeutischen Unternehmers keine personenbezogenen, insbesondere patientenbezogenen Daten enthalten dürfen.

\subsubsection{Fazit}

Die datenschutzrechtlichen Regelungen in den $\mathbb{s} 4$ off. AMC dürften auch für die Sekundärnutzung der bei klinischen Prüfungen im Sinne des Arzneimittelgesetzes gewonnenen personenbezogenen Daten gelten. Für die Sekundärnutzung von Arzneimitteltherapiedaten aus anderen Kontexten, seien es reine Heilversuche oder reguläre Behandlungen, werden durch das AMG jedoch keine besonderen Regelungen getroffen, weshalb es insoweit bei den im Übrigen anwendbaren Regeln bleibt, insbesondere also dem BDSG oder den Landeskrankenhausgesetzen sowie der ärztlichen Schweigepflicht.

\subsection{Länderspezifische Regelungen bei Unterbringung psychisch Kranker}

Jedes Bundesland verfügt über ein Gesetz, das die sogenannte öffentlich-rechtliche Unterbringung psychisch Kranker ermöglicht, wenn diese für sich oder andere eine nicht unerhebliche Gefahr darstellen. ${ }^{235}$ Neben der zwangsweisen Unterbrin-

235 Diese öffentlich-rechtliche bzw. (genauer) verwaltungsrechtliche Unterbringung ist von einer solchen auf strafrechtlicher Grundlage, dem sogenannten Maßregelvollzug, zu unterscheiden. Die Voraussetzungen für die Anordnung des Maßregelvollzugs durch Strafurteil (verkürzt: eingeschränkte oder fehlende Schuldfähigkeit) finden sich im StGB (s.u. S. 195, Fn. 619). Die Rahmenbedingungen der Durchführung des Maßregelvollzugs werden jedoch von den Bundesländern festgelegt. Teilweise finden deren Gesetze, welche die originär verwaltungsrechtliche Unterbringung regeln, entsprechend auch auf den Maßregelvollzug Anwendung (so nach $§ 15$ Abs. 1 UBG BW); im Übrigen bestehen eigene Gesetze für den Maßregelvollzug (so in Hamburg und im Saarland), welche aber inhaltlich den Unterbringungsgesetzen sehr ähneln. 
gung können diese Gesetze unter engen Voraussetzungen auch eine Zwangsbehandlung erlauben. Dies ist der Fall, wenn der Untergebrachte krankheitsbedingt zur Einsicht in den Behandlungsbedarf oder zum Handeln entsprechend dieser Einsicht nicht fähig ist, die Maßnahmen als letztes Mittel Besserung der Krankheit erfolgversprechend und für den Betroffenen nicht mit Belastungen verbunden sind, die außer Verhältnis zum zu erwartenden Nutzen stehen; außerdem sind besondere verfahrensmäßige Sicherungen zum Grundrechtsschutz geboten. ${ }^{236}$ Auch für eine freiwillige Behandlung im Rahmen einer zwangsweisen Unterbringung sind einschlägige Regelungen zum Datenumgang in den jeweiligen Gesetzen zu beachten.

Bezeichnet werden diese Gesetze entweder als Psychisch-Kranken-Gesetze (PsychKG), Freiheitsentziehungsgesetze (FreihEntzG) oder Unterbringungsgesetze (UBC). ${ }^{237}$ In manchen Bundesländern finden sich im jeweiligen Gesetz ausdrückliche Regelungen zum Datenschutz oder sogar Forschungsklauseln. In anderen Landesgesetzen fehlen solche Regelungen.

\subsubsection{Länder ohne spezifische Regeln}

Die Gesetze von Baden-Württemberg (UBC BW), Bayern (UnterbrC BY), Berlin (PsychKC BE), Hessen (FreihEntzG HE), Nordrhein-Westfalen (PsychKG NW), Saarland (UBG SL), Sachsen (PsychKG SN) und Sachsen- Anhalt (PsychKG ST) enthalten keine Regelungen zum Datenschutz, sodass es in Bezug auf den Umgang mit personenbezogenen Daten von psychisch Kranken in öffentlich-rechtlicher Unterbringung bei der Anwendung der allgemeinen datenschutzrechtlichen Normen bzw. - soweit anwendbar ${ }^{238}$ - der jeweiligen Normen der Landeskrankenhausgesetze bleibt.

\subsubsection{Sonderregeln zum Datenschutz}

Die Bundesländer Brandenburg (PsychKG BB), Bremen (PsychKG HB), MecklenburgVorpommern (PsychKG MV), Niedersachsen (PsychKG NI), Schleswig-Holstein (PsychKG SH) und Thüringen (PsychKG TH) haben in ihre Unterbringungsgesetze eigene Regelungen zum Datenschutz aufgenommen.

Grundsätzlich ordnen diese Landesunterbringungsgesetze die Geltung der jeweiligen Landesdatenschutzgesetze oder Landeskrankenhausgesetze an, soweit nichts Abweichendes im Unterbringungsgesetz geregelt wird. ${ }^{239}$ Für die mögliche Sekundärnutzung von Behandlungsdaten, die im Rahmen der öffentlich-rechtlichen Unterbringung psychisch Kranker generiert werden, sind folgende gesetzliche Maßgaben zu beachten:

236 BVerfG, Beschl. v. 12.10.2011 - 2 BvR 633/11, NJW 2011, 3571, zum UBG BW, welches in der damaligen Fassung diese Vorausset zungen nicht erfüllte, daher z.T. verfassungswidrig war und nur eine Zwangsunterbringung rechtfertigen konnte; entsprechend bereits BVerfG, Beschl. v. 23.03.2011 - 2 BvR 882/09, NJW 2011, 2113, zum damaligen Maßregelvollzugsgesetz RP. Die Zwangsunterbringung schließt für sich genommen die Freiwilligkeit einer ggf. erfolgten Behandlung nicht aus, wobei jedoch an die Feststellung der Freiwilligkeit hier besonders hohe Anforderungen zu stellen sind (vgl. BVerfG, Beschl. v. 23.03.2011, Rdnr. 41).

237 Vgl. Huster/Kaltenborn, Krankenhausrecht, $\ 16$ F Rdnr. 72.

238 Die psychiatrischen Unterbringungskliniken nehmen hier möglicherweise z.T. wie Maßregelvollzugskliniken eine Sonderstellung ein. So schließt z.B. das Saarland die Anwendung des LKHG SL auf die dortige Maßregelvollzugsklinik explizit aus (s.u. S. 195).

239 Vgl. § 54 PsychKG BB, § 46 PsychKG HB, § 43 PsychKG MV, § 32 PsychKG NI, § 27 PsychKG SH und § 36 PsychKG TH. 


\subsubsection{Brandenburg}

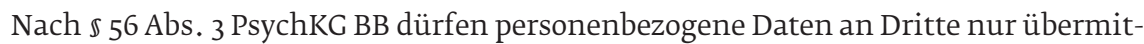
telt werden, wenn die betroffene Person eingewilligt hat, die Daten zur rechtmäßigen Aufgabenerfüllung dieser Stellen zwingend erforderlich sind und die Übermittlung zur Erfüllung von in diesem Gesetz, insbesondere in den $\mathbb{\$} \mathbb{5} 5,6,12,13,17,18$, 52 oder $\mathbb{} 53$ PsychKG BB, genannten Zwecken erfolgt oder die Übermittlung durch andere Rechtsvorschriften ausdrücklich zugelassen ist.

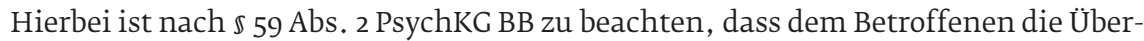
mittlung personenbezogener Daten mitzuteilen ist, sofern nicht schwerwiegende Gründe dafür sprechen, dass aufgrund dieser Mitteilung eine gegenwärtige erhebliche Gefahr für seine Gesundheit oder für die öffentliche Sicherheit entsteht.

\subsubsection{Bremen}

$\$ 47$ Abs. 1 PsychKC HB schränkt die Ausnahmen in Bezug auf die Zweckbindung der Daten nach $\mathbb{3} 32$ Abs. 2 des Bremer Gesundheitsdienstgesetzes insoweit ein, als dass eine Verarbeitung oder Übermittlung personenbezogener Daten nur dann zulässig ist, wenn (1) der oder die Betroffene eingewilligt hat oder (2) wenn eine gegenwärtige Gefahr für Leib oder Leben der betroffenen Person oder Dritter nicht anders abgewendet werden kann.

\subsubsection{Mecklenburg-Vorpommern}

S 43 PsychKC MV enthält zwar Regelungen zum Datenschutz, allerdings keine Sondervorschriften in Bezug auf die Sekundärnutzung von Behandlungsdaten, so dass insoweit Landeskrankenhaus bzw. Landesdatenschutzgesetz gelten ( $\$ 43 \mathrm{Abs} .1$ PsychKG MV). ${ }^{240}$

\subsubsection{Niedersachsen}

$\mathbb{S} 33$ Abs. 1 PsychKG NI ordnet an, dass personenbezogene Daten, die einem Berufsoder besonderen Amtsgeheimnis unterfallen, für andere Zwecke als die, für die die Daten erhoben oder erstmals nach $₫ 10 \mathrm{Abs}$. 1 S. 2 LDSG NI gespeichert worden sind, nur gespeichert, verändert, übermittelt oder sonst genutzt werden dürfen, wenn (1) die betroffene Person eingewilligt hat, (2) ein Gesetz dies vorschreibt oder (3) eine Lebensgefahr oder eine Gefahr für die körperliche Unversehrtheit nicht anders abgewendet werden kann. Werden Daten übermittelt, so hat der Empfänger diese Daten gegen unbefugte Kenntnisnahme zu sichern, worauf der Empfänger hinzuweisen ist, $\mathbb{3} 3$ Abs. 2 PsychKG NI.

Besonders schutzwürdige Daten, solche, die einem Berufs- oder besonderen Amtsgeheimnis unterfallen ( $\$ 33$ Abs. 1 S. 1 PsychKG NI), dürfen nur gespeichert werden, soweit dies für die Erfüllung der in diesem Gesetz vorgesehenen Aufgaben oder für die Dokumentation von diagnostischen oder therapeutischen Maßnahmen erforderlich ist, $\mathbb{} 35$ Abs. 1 PsychKG NI.

240 Zur Abgrenzung von LKHG MV zu LDSG MV s.u. S. 172ff., wobei zu beachten ist, dass die Unterbringungskliniken öffentliche Stellen sind oder aufgrund hoheitlicher Beleihung zumindest als solche gelten. Auch kann man aufgrund der zwangsweisen Unterbringung nicht von einer Teilnahme am Wettbewerb um Patienten ausgehen. 


\subsubsection{Schleswig-Holstein}

Die $\$ \$ 28$ und 30 PsychKG SH treffen eine inhaltlich nahezu identische Regelung wie die der $\$ \$ 33$ und 35 PsychKG NI.

\subsubsection{Thüringen}

$\mathbb{\$} \$ 36$ bis 38 PsychKG TH enthalten zwar Regelungen zum Datenschutz, allerdings keine Sondervorschriften in Bezug auf die Sekundärnutzung von Behandlungsdaten, so dass insoweit Landeskrankenhaus bzw. Landesdatenschutzgesetz gelten $(\mathbb{S} 36$ Abs. 1 PsychKG TH).

\subsubsection{Sonderregelungen zur Forschung mit personenbezogenen Daten}

Hamburg und Rheinland-Pfalz haben in ihren Unterbringungsgesetzen explizite Regelungen im Hinblick auf den Umgang mit personenbezogenen Daten zu Forschungszwecken.

\subsubsection{Hamburg}

$\mathbb{} 31$ PsychKG HH regelt die Forschung mit personenbezogenen Daten. Demnach gilt für die Verarbeitung der nach $\mathbb{2} 27$ PsychKG HH erhobenen und der nach $\mathbb{\$} 28$ PsychKC HH gespeicherten personenbezogenen Daten für Forschungszwecke $\mathbb{} 27$ LDSG HH mit folgenden Maßgaben: Eine Übermittlung an nicht-öffentliche Stellen ist nur zulässig, wenn der Betroffene eingewilligt hat oder die Daten vor der Übermittlung so verändert werden, dass ein Bezug auf eine bestimmte natürliche Person nicht mehr erkennbar ist. Über die Übermittlung entscheidet die für das Gesundheitswesen zuständige Behörde.

\subsubsection{Rheinland-Pfalz}

\$35 PsychKG RP modifiziert den Datenschutz bei Forschungsvorhaben. So dürfen die mit der Durchführung von Hilfen, Schutzmaßnahmen und Unterbringungen befassten Ärzte die bei ihnen in diesem Zusammenhang anfallenden personenbezogenen Daten für eigene wissenschaftliche Forschungsvorhaben speichern und nutzen. Dies gilt entsprechend für sonstiges wissenschaftliches Personal, soweit es der Geheimhaltungspflicht des $\$ 203$ des Strafgesetzbuches unterliegt ( $\$ 35$ Abs. 1 S. 1 und 2 PsychKG RP). Zu Zwecken der wissenschaftlichen Forschung ist die Übermittlung von personenbezogenen Daten, die im Zusammenhang mit der Durchführung von Hilfen, Schutzmaßnahmen und Unterbringungen anfallen, an Dritte und die Speicherung und Nutzung durch sie zulässig, wenn die betroffene Person eingewilligt hat ( $\$ 35$ Abs. 2 PsychKG RP). Hierbei gelten $\mathbb{3} 34$ Abs. 2 Sätze 2 bis 4 PsychKG RP, in denen die Anforderungen an die Einwilligung statuiert werden, entsprechend. Nach $\mathbb{} 35$ Abs. 3 PsychKG RP sind die personenbezogenen Daten zu anonymisieren, sobald dies nach dem Forschungszweck möglich ist. Solange dies nicht möglich ist, sind die Merkmale, mit deren Hilfe ein Personenbezug hergestellt werden kann, gesondert zu speichern, sobald es der Forschungszweck erlaubt; die Merkmale sind zu löschen, sobald der Forschungszweck erreicht ist. An Personen und Stellen, auf die die Bestimmungen dieses Gesetzes keine Anwendung finden, dürfen nach $\mathbb{} 35$ Abs. 4 
PsychKG RP personenbezogene Daten nur übermittelt werden, wenn sie sich verpflichten, (a) die Daten nur für das von ihnen genannte Forschungsvorhaben zu verwenden, (b) die Bestimmungen des Abs. 3 einzuhalten und (c) dem Landesbeauftragten für den Datenschutz und die Informationsfreiheit auf Verlangen Einsicht und Auskunft zu gewähren, sowie wenn sie nachweisen, dass bei ihnen die technischen und organisatorischen Voraussetzungen vorliegen, um die Verpflichtung nach Buchst. b zu erfüllen. 